\title{
Avaliação e recomendações nutricionais para crianças e adolescentes com fibrose cística
}

\section{Nutritional assessment and recommendations for children and adolescents with cystic fibrosis}

\author{
Célia Regina M. M. Chaves ${ }^{1}$, Ana Lucia P. Cunha ${ }^{2}$
}

\section{RESUMO}

Objetivo: Revisar e discutir evidências sobre a avaliação do estado nutricional e as recomendações para o tratamento nutricional de crianças e adolescentes com fibrose cística.

Fontes de dados: Bancos de dados MEDLINE (versão PubMed) e Latin American and Caribbean Center on Health Sciences Information (LILACS), entre 1984 a 2009. Aplicou-se a combinação dos seguintes descritores: fibrose cística, estado nutricional, criança e adolescente - em inglês e português.

Síntese dos dados: A fibrose cística é uma doença genética, de evolução crônica, progressiva e fatal. Resulta do defeito na proteína reguladora transmembrana que regula a condução de cloro e, consequentemente, o fluxo de sódio e água através da membrana apical das células epiteliais. Pacientes fibrocísticos são vulneráveis à desnutrição, que resulta do desequilíbrio entre ingestão alimentar, gasto e perdas energéticas. Novos conhecimentos sobre a fisiopatologia da doença, adquiridos nas últimas décadas, resultaram em mudanças significativas nas atuais recomendações energéticas e principalmente de lipídeos. A importância da nutrição no bem-estar e sobrevida dos fibrocísticos está bem estabelecida, assim como a associação entre a desnutrição e a deterioração da função pulmonar. Existem múltiplos fatores inter-relacionados que afetam o estado nutricional, tais como, mutação genética, insuficiência pancreática, ressecção intestinal, perda de sais e ácidos biliares, refluxo gastroesofágico, inflamação e infecções pulmonares, diabetes e condições emocionais.

Conclusões: $\mathrm{O}$ monitoramento nutricional e o aconselhamento dietético são elementos chave no manejo de crianças e adolescentes com fibrose cística com o intuito de controlar a sintomatologia e a progressão da doença, proporcionando melhor qualidade de vida.

Palavras-chave: fibrose cística; estado nutricional; criança; adolescente.

\section{ABSTRACT}

Objective: To review and discuss evidence on the nutritional status assessment and recommendations for nutritional management of children and adolescents with cystic fibrosis.

Data sources: MEDLINE (PubMed version) and Latin American and Caribbean Center on Health Sciences Information (LILACS), from 1984 to 2009. The combination of the following keywords were applied: cystic fibrosis, nutritional status, child, and adolescent.

Data Synthesis: Cystic fibrosis (CF) is a genetic disease, chronic, progressive, and fatal. It is caused by a defect in the transmembrane regulatory protein that regulates the flow of chlorine, sodium and water across the apical membrane of epithelial cells. CF patients are vulnerable to malnutrition resulting from the imbalance between food intake, energy expenditure and loss. New insights into the pathophysiology of the disease have resulted in significant changes in current recommendations for energy and especially lipids intake. There are many interrelated factors that affect the nutritional status of CF patients, such as genetic mutation, pancreatic insufficiency, bowel resection, loss of bile salts and acids, gastroesophageal reflux disease, lung inflammation and infections, diabetes, and emotional conditions. The importance of nutrition in the survival of CF patients is well
Instituição: Instituto Fernandes Figueira, Rio de Janeiro, RJ, Brasil ${ }^{1}$ Doutora em Clínica Médica pela Universidade Federal do Rio de Janeiro (UFRJ); Chefe do Departamento de Nutrição e Alimentação do Instituto Fernandes Figueira, Rio de Janeiro, RJ, Brasil

2Doutoranda em Ciências Nutricionais pela UFRJ; Nutricionista do Departamento de Nutrição e Alimentação do Instituto Fernandes Figueira, Rio de Janeiro, RJ, Brasil
Endereço para correspondência:

Célia Regina M. M Chaves

Av. Rui Barbosa 716 - Flamengo

CEP 22250-020 - Rio de Janeiro/RJ

E-mail: crchaves@iff.fiocruz.br

Conflito de interesse: nada a declarar 
established, as well as the association between malnutrition and deterioration of lung function.

Conclusions: Nutritional monitoring and dietary counseling are key elements in the management of patients with $\mathrm{CF}$ aiming to control the symptoms and the progression of the disease in order to provide better quality of life.

Key-words: cystic fibrosis; nutritional status; child; adolescent.

\section{Introdução}

A fibrose cística (FC) é uma doença genética de herança autossômica recessiva, mais frequente na população caucasóide, de evolução crônica, progressiva e, na maioria das vezes, fatal $^{(1)}$. A incidência é de 1:2.000 a 2.500 nascimentos na raça branca, diminuindo para 1:17.000 entre os negros ${ }^{(2)}$. Ela é causada pelo defeito no gene responsável pela codificação da proteína reguladora de condutância transmembrana da fibrose cística (Cystic Fibrosis Transmembrane Regulator-CFTR), que regula o transporte do íon cloreto de células epiteliais da árvore respiratória, trato digestório, glândulas sudoríparas e aparelho reprodutor ${ }^{(3)}$. As manifestações clássicas da FC são: doença pulmonar obstrutiva supurativa crônica, insuficiência pancreática, níveis elevados de eletrólitos no suor e infertilidade masculina ${ }^{(4)}$.

O prognóstico e a expectativa de vida dos fibrocísticos melhoraram muito nos últimos anos e o tratamento nutricional contribuiu para tais resultados ${ }^{(5)}$. O estado nutricional é considerado um agente independente determinante na evolução da doença e estes pacientes frequentemente são desnutridos $^{(6)}$. Entretanto, alcançar e manter um estado nutricional adequado é um desafio. Assim o objetivo deste artigo foi revisar e discutir evidências sobre a avaliação do estado nutricional e as recomendações para o tratamento nutricional de crianças e adolescentes com fibrose cística.

A detecção da desnutrição deve ser realizada mediante a combinação de diferentes métodos em função da disponibilidade dos mesmos. Segundo Groeneweg et $a l^{(7)}$, a avaliação da massa magra é importante no diagnóstico nutricional dos fibrocísticos. Associada à diminuição da musculatura esquelética respiratória e do surfactante pulmonar, à depressão do sistema imunológico e à alteração da arquitetura pulmonar, a redução da massa magra predispõe à infecção e ao aumento do gasto energético ${ }^{(7)}$.

Um crescimento satisfatório dos três aos seis anos de idade é preditivo de melhor função pulmonar, reduzindo a morbidade e a mortalidade ${ }^{(8)}$. A adequação do peso expressa em percentual do peso ideal mostrou associação com a função pulmonar ${ }^{(9)}$. Em crianças, a eutrofia está relacionada ao sucesso do tratamento e corresponde ao percentil maior ou igual ao 25 nas curvas de crescimento, segundo os Consensos de Nutrição na FC, que são mais rigorosos quanto aos pontos de corte. O Consenso Americano recomenda avaliar a adequação, o ganho de peso e o ritmo de crescimento linear ${ }^{(9)}$. Quando o parâmetro utilizado é o percentual de adequação, devem-se considerar valores superiores a $90 \%{ }^{(3,9)}$. Entretanto, a orientação do Subcomitê de Nutrição e Crescimento da Fundação de FC recomenda que pacientes de dois a 20 anos mantenham o percentil de índice de massa corporal (IMC) igual ou maior que 50 para sexo e idade ${ }^{(9,10)}$.

\section{Metabolismo energético na fibrose cística}

O balanço energético depende do equilíbrio entre a ingestão e o gasto com metabolismo basal, crescimento e perdas. A taxa metabólica basal (TMB) elevada, encontrada nestes pacientes, pode ser causada pelo defeito intrínseco no metabolismo energético, presente em determinadas mutações genéticas, aumentando a utilização de energia com maior gasto de adenosina trifosfato $^{(11-13)}$.

O principal mecanismo do aumento do gasto energético pode ser atribuído à elevação das concentrações séricas de catecolaminas e à liberação de citocinas durante a resposta inflamatória pulmonar. Deste modo, à medida que a função pulmonar piora, a TMB aumenta ${ }^{(11,13,14)}$.

As principais causas da perda energética são a má digestão e a má absorção intestinal, levando à perda de gordura pelas fezes, decorrente principalmente da insuficiência pancreática exócrina ${ }^{(8)}$. A redução da ingestão energética ocorre devido à anorexia, causada por liberação de citocinas - fator de necrose tumoral (TNF) e interleucinas (IL) - durante a inflamação e a infecção pulmonar aguda e crônica. Além da depressão emocional, os fibrocísticos apresentam diminuição do paladar pela deficiência de zinco, aumento da secreção pulmonar, dispneia, esofagite causada pelo refluxo gastroesofágico e iatrogenia, quando há restrição lipídica na alimentação. Outros fatores que também contribuem para esse déficit energético são: glicosúria, devido ao diabetes melito (DM) por falência do pâncreas endócrino, e os vômitos resultantes do refluxo gastroesofágico $(\mathrm{RGE})^{(15)}$. 


\section{Metabolismo lipídico na fibrose cística}

O uso adequado das enzimas é fundamental para a correção da esteatorreia na FC. Deve-se observar a dose e o horário de administração, aderência ao tratamento e a forma de administração, ou seja, se a cápsula é ingerida fechada e sem mastigar. No caso dos lactentes, a administração pode ser com suco de maçã ou pequena quantidade de leite materno ou artificial. As refeições devem ser realizadas em bloco para aproveitar toda enzima administrada e os beliscos entre as refeições desestimulados ${ }^{(16)}$.

A deficiência de ácidos graxos essenciais (AGE) encontrada nestes pacientes era atribuída à má digestão e má absorção lipídica, com desnutrição e aumento da $\beta$-oxidação de ácidos graxos poliinsaturados ${ }^{(17)}$. Entretanto, a baixa concentração de ácido linoléico $(\mathrm{AL})$ também foi observada em pacientes bem nutridos que recebiam uma dieta adequada em lipídeos e não apresentavam má absorção de gordura, devido à reposição enzimática regular ${ }^{(18,19)}$. Por isso, a falha na regulação do metabolismo destes ácidos graxos parece estar relacionada ao defeito funcional da CFTR, que reduz a incorporação do AL nos fosfolipídeos da membrana celular ${ }^{(20)}$.

Entre os AGE que mais comumente encontram-se deficientes na FC, estão o AL e o ácido docosahexaenoico (DHA), representantes das séries n-6 e n-3, respectivamente. Em contraste, as concentrações séricas do ácido araquidônico (AA) estão geralmente preservadas ou até mesmo elevadas ${ }^{(21,22)}$. A conversão de AL nos metabólitos da série n-6, como o AA, está desregulada na $\mathrm{FC}^{(23)}$.

A manutenção de concentrações séricas adequadas de AGE está associada com melhores índices de crescimento em crianças com $\mathrm{FC}^{(21,24)}$. Embora o aumento do consumo energético seja importante para o ganho de peso e recuperação do crescimento, esse efeito é potencializado quando ocorre aumento da ingestão do AL e, consequentemente, o aumento de sua concentração plasmática ${ }^{(24)}$. A suplementação apenas com AL pode favorecer o aumento do AA e da inflamação ${ }^{(25)}$.

O desequilíbrio do metabolismo lipídico resulta no aumento da produção de eicosanoides, como as prostaglandinas e leucotrienos das séries 2 e 4, desencadeando o mecanismo responsável pela inflamação na $\mathrm{FC}$ e o desenvolvimento precoce da doença pulmonar ${ }^{(26)}$. Por outro lado, o processo inflamatório aumenta a liberação de radicais livres com peroxidação dos lipídeos das membranas celulares, o que justifica a reposição adequada de AGE e antioxidantes ${ }^{(27)}$.

Alguns autores observaram que a suplementação com óleo de peixe, que contém ácidos graxos da série n-3 (eicosapentaenoico - EPA e DHA) restaura o desequilíbrio desses ácidos graxos nos fosfolipídeos das membranas celulares e reduz os níveis séricos de triglicerídeos, imunoglobulina $\mathrm{G}(\mathrm{IgG})^{(25)}$ e mediadores inflamatórios (TNF- $\alpha$ e IL- 1$)^{(23)} \mathrm{e}$ diminui a razão entre leucotrienos da serie 4 e $5\left(\mathrm{LTB}_{4} / \mathrm{LTB}_{5}\right)^{(27)}$. A ingestão de DHA pode reduzir a inflamação crônica na FC por meio da regulação da produção dos eicosanoides derivados do $\mathrm{AA}^{(27)}$. Entretanto, são necessários mais estudos controlados e randomizados para determinar os efeitos terapêuticos da suplementação dos AG n-3 na FC ${ }^{(28)}$.

\section{Recomendações nutricionais}

O tratamento nutricional deverá ser estabelecido após a avaliação antropométrica e do consumo alimentar do paciente. Para isso, utiliza-se preferencialmente o registro alimentar de três ou cinco dias ${ }^{(9,27)}$.

Em crianças com FC, o gasto energético total (GET) é 25 a $27 \%$ maior do que o esperado de acordo com a idade e sexo para crianças saudáveis ${ }^{(3)}$ e pode, dependendo do caso, chegar a $200 \%$ da recomendação da Dietary Reference Intakes (DRI) ${ }^{(3,9,10,24,29)}$. O cálculo da necessidade energética total deve ser realizado pela estimativa da taxa do metabolismo basal, recomendada pela Organização Mundial da Saúde (OMS), multiplicada pelo fator atividade mais o coeficiente da doença pulmonar, baseado no volume expiratório final do primeiro segundo $\left(\mathrm{VEF}_{1}\right)$, multiplicado pelo coeficiente de absorção de gordura fecal $^{(29-31)}$ (Tabela 1). O cálculo da necessidade energética é difícil mesmo em pessoas saudáveis e, na clínica diária, nem sempre é possível dispor desses três valores. Além disso, há uma grande variação intra-individual no que diz respeito ao crescimento e ingestão energética ${ }^{(24)}$. Por isso, é recomendado que o ajuste do gasto energético seja feito a partir da avaliação do ganho de peso, da reserva adiposa e da manutenção do crescimento ${ }^{(9)}$. Entretanto, essas fórmulas devem ser utilizadas como ponto inicial para o tratamento e naqueles com pouco desenvolvimento e crescimento. Segundo Wilson et $a l^{(31)}$, o metabolismo basal e o gasto energético total são normais em crianças assintomáticas.

A distribuição dos macronutrientes em percentual do valor energético total deve ser aproximadamente de 15 a $20 \%$ de proteínas, 40 a $50 \%$ de carboidratos e 35 a $40 \%$ de lipídeos. A ingestão proteica pode chegar a 150-200\% do recomendado de acordo com a idade e sexo para crianças saudáveis, sendo $2 / 3$ dessa proteína de alto valor biológico ${ }^{(9,10)}$.

É fundamental desmistificar a restrição de gorduras na alimentação do fibrocístico. A comparação da sobrevida 
dos pacientes de Boston e Toronto demonstrou aumento significativo da sobrevida dos últimos após a prescrição de dieta hipercalórica sem restrição de gordura ${ }^{(31)}$. A maior quantidade de lipídeos aumenta o valor energético e a palatabilidade da refeição propiciando a elevação da ingestão energética total ${ }^{(30,32)}$. A distribuição de lipídeos deve conter menos de $10 \%$ de ácidos graxos saturados, menos de $1 \%$ de trans, menos de $10 \%$ de poliinsaturados e o restante de monoinsaturados ${ }^{(33,32)}$.

Os triglicerídeos de cadeia média estão recomendados em condições especiais, como na síndrome do intestino curto, doença hepática e em pacientes recebendo altas doses de enzima apesar das condutas para tentar diminuir a esteatorreia $^{(12)}$. A recomendação de AL e ácido linolênico é de 5 e $10 \%$ respectivamente da ingestão energética total; sendo que, do total dos ácidos graxos n-3, 10\% devem ser ingeridos na forma de EPA e $\mathrm{DHA}^{(34)}$.

Os lactentes apresentam um alto requerimento energético ${ }^{(35)}$. O leite humano deve ser oferecido por conter nutrientes que auxiliam a digestão, crescimento e função imunológica. Entretanto, seu uso necessita de monitoramento cuidadoso de proteínas e eletrólitos séricos devido a duas complicações metabólicas: hipoproteinemia e alcalose hiponatrêmica. A complementação com fórmula artificial é necessária no caso de dificuldades no ganho ponderal e no aporte proteico. $\mathrm{O}$ aumento da densidade calórica das fórmulas pode ser alcançado com adição de 10 a $12 \%$ de polímeros de glicose e $5 \%$ de gorduras ${ }^{(33)}$. A fórmula polimérica é muito bem aceita, reserva-se a hidrolisada para casos de má absorção resultante de intestino curto secundário a cirurgia do trato intestinal ou alergia ao leite de vaca ${ }^{(12)}$.
$\mathrm{Na}$ doença pulmonar avançada, as refeições devem ser fracionadas em pequenos volumes para evitar o aumento do gasto de oxigênio, retificação do diafragma e hipermetabolismo. A dieta deve ser normocalórica e normoproteica para não aumentar o estímulo respiratório. O uso de fórmulas enterais especializadas para pacientes com doença pulmonar é controverso. As fórmulas hiperlipídicas indicadas podem apresentar algumas desvantagens, como o desconforto abdominal, diarreia, retardo no esvaziamento e aumento do resíduo gástrico, o que é um risco para aspiração pulmonar. Sendo assim, deve haver uma oferta equilibrada dos macronutrientes, incluindo os carboidratos, que são fonte de energia primária para a musculatura respiratória ${ }^{(36)}$.

\section{Intervenção nutricional}

Além da estimativa do gasto, existe também uma grande dificuldade em atingir o requerimento energético. Segundo Powers et $a^{(37)}$, o acréscimo de energia deve ser gradual e associado a orientações para mudança de comportamento da criança e da família. Neste período de adaptação, a criança e a família devem ter um acompanhamento mais próximo (semanal) e não restrito ao calendário de uma consulta a cada três meses, como os consensos recomendam.

A análise e a evolução do IMC, peso para estatura (P/E) e estatura para idade (E/I) permitem identificar o paciente em falência ou risco nutricional ${ }^{(28)}$, conforme demonstrado na Tabela 2.

De acordo com o Consenso Europeu, a indicação do suporte nutricional é para pacientes com P/E de 85 a $90 \%$ do peso ideal (PI), falha em responder à dieta hipercalórica, com $\mathrm{P} / \mathrm{E}$ caindo

Tabela 1 - Fórmulas para estimar as necessidades energéticas de crianças e adolescentes com fibrose cística - adaptado de Oliveira e Oliveira(33)

\begin{tabular}{ccc}
\hline \multicolumn{2}{c}{ GET=GER x (fator atividade + coeficiente da doença) x coeficiente de absorção de gordura fecal } \\
\hline Gasto energético em repouso (fórmulas da Organização Mundial da Saúde) \\
\hline Idade (anos) & Masculino & Feminino \\
\hline $0-3$ & $60,9 \times \mathrm{P}-54$ & $61 \times \mathrm{P}-51$ \\
$3-10$ & $22,7 \times \mathrm{P}-495$ & $22,5 \times \mathrm{P}-499$ \\
$10-18$ & $17,5 \times \mathrm{P}+651$ & $12,2 \times \mathrm{P}+746$ \\
\hline Fator atividade & Coeficiente da doença (VEF ${ }_{1}$ ) & Coeficiente de absorção de gordura fecal (CAGF) \\
\hline Acamado=1,3 & Grave: $\leq 40 \%=0,3$ & Normal: $\geq 93 \%=0$ \\
Sedentário=1,5 & Moderada: $40-79 \%=0,2$ & \\
Ativo=1,7 & Normal: $\geq 80 \%=0$ & \\
\hline *Determinado pela fração da ingestão de gordura (exemplo: absorção de $80 \%=0,93 / 0,80=1,16)$ & \\
Caso não disponha do resultado da gordura fecal, utilizar valor de aproximadamente 0,85 & \\
\hline
\end{tabular}

GET: gasto energético total; GER: gasto energético de repouso; P: peso; VEF : volume expiratório final do primeiro segundo; CAGF: coeficiente de absorção de gordura fecal 
para $85 \%$ ou deterioração da função pulmonar ${ }^{(3)}$. Rosenfeld et a ${ }^{(32)}$ também consideram o $\mathrm{P} / \mathrm{E}$ estagnado por três meses e a E/I menor que o percentil 5 como critério para suplementação calórica. Segundo estes autores a nutrição enteral em longo prazo melhora o crescimento e a composição corporal.

Entretanto, segundo McDonald ${ }^{(38)}$ a ferramenta de rastreamento nutricional desenvolvida pelo Consenso Europeu de 2002 para a classificação das categorias de risco apresenta algumas ambiguidades e nenhum estudo validando esta ferramenta foi publicado. Além disso, há o efeito psicológico no indivíduo intitulado de portador de falência nutricional. Assim, McDonald ${ }^{(38)}$, criou um instrumento de rastreamento de risco nutricional baseando-se no percentil de IMC, ganho ponderal por dia e estatura anual (Tabela 3). Como muitas crianças são diagnosticadas após apresentarem alteração no desenvolvimento pôndero-estatural, os ganhos de peso e estatura acompanhados longitudinalmente são mais indicativos de alteração do estado nutricional do que o percentil absoluto de peso e estatura para idade. Considera-se o crescimento como normal quando o peso e a estatura estão entre o percentil 10 e 50 . O ganho de peso esperado deverá ser maior quando o percentil for menor (no percentil 10, deverá ser maior do que para o percentil 50). Para validar seu instrumento, McDonald ${ }^{(38)}$ comparou-o com a recomendação do Consenso Europeu e obteve sensibilidade e especificidade de 84 e $75 \%$, respectivamente.

A intervenção nutricional deve ser a princípio com alimentos de alta densidade calórica. Entretanto, existem pacientes que mesmo com o uso destes alimentos e da reposição enzimática correta não conseguem manter um bom estado nutricional. Nestes casos, é necessária a introdução de suplementos energéticos administrados por via oral, enteral ou ostomia ${ }^{(10,12,39)}$. Para a suplementação calórica por via oral não substituir as refeições usuais, recomenda-se sua administração nos intervalos das refeições e antes de dormir ${ }^{(21,40)}$. A baixa palatabilidade de algumas fórmulas enterais pode dificultar a adesão da via oral. Os resultados significantes da suplementação calórica são observados em pacientes desnutridos ou em períodos de perda aguda de peso, sendo pouco efetiva em casos de estado nutricional subótimo ${ }^{(39)}$.

Com relação à densidade energética, as fórmulas padrão $(1,0 \mathrm{kcal} / \mathrm{mL})$, ou seja, normocalóricas, geralmente são bem toleradas. As hipercalóricas $(1,5-2,0 \mathrm{kcal} / \mathrm{mL})$ também podem ser necessárias para assegurar a adequação energética ${ }^{(10)}$. A quantidade depende da necessidade de cada paciente. Segundo Arias et al ${ }^{(40)}$, a quantidade calórica suplementada varia com a idade, sendo $200 \mathrm{kcal}$ para menores de dois anos, $400 \mathrm{kcal}$ para préescolares, $600 \mathrm{kcal}$ para escolares e $800 \mathrm{kcal}$ para adolescentes.

Tabela 2 - Identificação do paciente em falência ou risco nutricional - adaptado de Borowitz, Baker e Stallings ${ }^{(9)}$

\begin{tabular}{lccccc}
\hline Estado nutricional & Estatura & \% PI/l & $\begin{array}{c}\text { P/E } \\
(\mathbf{0 - 2} \text { anos })\end{array}$ & $\begin{array}{c}\text { IMC } \\
(\mathbf{2 - 2 0 a n o s})\end{array}$ & Ação \\
\hline Aceitável & Normal & $>90 \%$ & $>\mathrm{p} 25$ & $\mathrm{p} 25$ & Manter conduta \\
Em risco & $\begin{array}{c}\text { Fora do potencial } \\
\text { genético }\end{array}$ & $\begin{array}{c}\text { Com perda de peso } \\
\text { ou peso mantido }\end{array}$ & $>\mathrm{p} 10 \mathrm{e}<\mathrm{p} 25$ & $>\mathrm{p} 10 \mathrm{e}<\mathrm{p} 25$ & $\begin{array}{c}\text { Alguns podem estar } \\
\text { em risco }\end{array}$ \\
Falência nutricional & $<\mathrm{p} 5$ & $<90 \%$ & $<\mathrm{p} 10$ & $<\mathrm{p} 10$ & Tratar falência \\
\hline
\end{tabular}

PI/I: peso ideal para idade; P/E: peso para estatura; IMC: índice de massa corporal; p: percentil

Tabela 3 - Rastreamento de risco nutricional baseando-se no percentil de índice de massa corporal, ganho ponderal por dia e estatural anual - adaptado de McDonald ${ }^{(38)}$

\begin{tabular}{lccc}
\hline & \multicolumn{2}{c}{ Escore do rastreamento de risco nutricional } \\
\hline & 0 ponto & 1 ponto & 2 pontos \\
\hline Percentil de IMC & $\geq 50 \%$ & $<50 \%$ e $\geq 10 \%$ & $<10 \%$ \\
Ganho ponderal diário & $\geq$ mínimo & $>0<$ mínimo & Perda ou falta de ganho ponderal \\
Ganho anual de estatura & $\geq$ mínimo & $>0<$ mínimo & Nenhum ganho na altura \\
\hline \multicolumn{2}{c}{ Pontos totais de risco nutricional/ Categorias do rastreamento de risco nutricional } \\
\hline $\begin{array}{l}\text { Pontuação do risco nutricional } \\
\text { Categoria do risco nutricional }\end{array}$ & Nenhum ou risco baixo & Risco moderado & $\geq 4$ \\
\hline
\end{tabular}

IMC: índice de massa corporal 
Quando as tentativas com dieta hipercalórica e suplementação oral são frustradas, há indicação de nutrição mais agressiva. Nestes casos, indica-se a sonda enteral para períodos curtos sem substituir a alimentação habitual. A infusão deve ser noturna, iniciada em ambiente hospitalar com um terço a metade das necessidades energéticas diárias, aumentando-se primeiro a densidade e depois o volume, conforme a evolução. É importante reforçar que a administração de enzimas deve ocorrer no início da infusão ${ }^{(9)}$. O tipo de fórmula - polimérica ou oligomérica - depende da evolução do paciente. Quando o período estimado para a suplementação calórica via enteral for longo, deve ser discutida com o paciente e a família a indicação da gastrostomia ${ }^{(33)}$. Os resultados obtidos com o seu uso são observados em períodos longos, geralmente superiores a três anos e, por isso, muitos estudos mostram respostas insuficientes em menor tempo de observação ${ }^{(32,40,41)}$.

Cabe ressaltar que a suplementação calórica deve ser avaliada pela melhora da composição corporal, com recuperação da massa magra corporal. A nutrição parenteral só é indicada nos casos em que o trato gastrointestinal não está funcionando nos pacientes muito desnutridos, em espera de transplante, ou quando não se consegue alcançar os requerimentos energéticos por via enteral ${ }^{(42)}$.

\section{Micronutrientes}

Os pacientes com FC apresentam estresse oxidativo elevado e deficiência de antioxidantes, sugerindo que a ativação constante do sistema imune contribui para a depleção de vitaminas e minerais, predispondo à ocorrência de danos celulares e piora do estado clínico ${ }^{(43)}$.

A absorção de vitaminas lipossolúveis (A, D, E e K) é deficiente em pacientes com esteatorreia e correlacionada com sua gravidade $^{(44)}$. Entretanto, a terapia de reposição enzimática não é suficiente para corrigir esse déficit, sugerindo outros mecanismos responsáveis pela carência. No caso da vitamina A, sugere-se um defeito na mobilização das reservas hepáticas. Em relação à vitamina $\mathrm{E}$, parece que a baixa disponibilidade de sais biliares na luz intestinal exerce uma influência igual ou mais importante na etiologia da sua deficiência do que a insuficiência pancreática. Por ser um poderoso antioxidante, a sua carência reduz a capacidade antioxidante dos lipídeos plasmáticos, sendo a única a ser suplementada nos pacientes com suficiência pancreática ${ }^{(45)}$.

A infecção e a inflamação crônica aumentam a produção de radicais livres. A redução do nível sérico de antioxidantes como das vitaminas $\mathrm{A}$ e E está associada à piora do quadro respiratório. Por outro lado, o aumento do estresse oxidativo parece aumentar o consumo dos nutrientes antioxidantes ${ }^{(26)}$, gerando um ciclo vicioso. A vitamina A ainda apresenta um papel importante na manutenção da integridade do epitélio respiratório e gastrointestinal ${ }^{(45)}$.

Com relação à vitamina $\mathrm{D}$, as baixas concentrações séricas da proteína ligadora de vitamina $\mathrm{D}$ são apontadas como a principal causa desta carência em pacientes homozigotos, sugerindo que a hipovitaminose $\mathrm{D}$ é basicamente um problema de transporte realizado por esta proteína carreadora. Outro fator contribuinte é a baixa exposição solar, principalmente em países frios ${ }^{(40)}$.

Após a descoberta da importância da vitamina $\mathrm{K}$ na saúde óssea, a atenção deixou de ser dispensada apenas para pacientes com distúrbios de coagulação, devido a sua ação na carboxilação dos resíduos glutâmicos das proteínas séricas (osteocalcina), na proteína óssea não colágena que apresenta alta afinidade pelo cálcio livre, aumentando sua biodisponibilidade ${ }^{(3)}$ As doses da suplementação diária das vitaminas lipossolúveis são definidas de acordo com a faixa etária (Tabela 4$)^{(45)}$.

Tabela 4 - Recomendação diária de vitaminas lipossolúveis e minerais e elementos traços na fibrose cística de acordo com a faixa etária - adaptado de Borowitz Baker e Stallings ${ }^{(9)}$

\begin{tabular}{|c|c|c|}
\hline Nutriente & Idade (anos) & $\begin{array}{c}\text { Recomendação de } \\
\text { ingestão/dia }\end{array}$ \\
\hline \multirow[t]{4}{*}{ Vitamina A } & $<1$ & 1500UI \\
\hline & 1 a 3 & $5000 \mathrm{UI}$ \\
\hline & 4 a 8 & 5000 a $10000 \mathrm{U}$ \\
\hline & $>8 a$ & 10000 \\
\hline \multirow[t]{4}{*}{ Vitamina D } & $<1$ & 400mg/dia \\
\hline & 1 a 3 & 400 a $800 \mathrm{mg} / \mathrm{dia}$ \\
\hline & 4 a 8 & 400 a $800 \mathrm{mg} / \mathrm{dia}$ \\
\hline & $>8$ & 400 a $800 \mathrm{mg} / \mathrm{dia}$ \\
\hline \multirow[t]{4}{*}{ Vitamina $\mathrm{E}$} & $<1$ & 40 a $50 \mathrm{mg} / \mathrm{dia}$ \\
\hline & 1 a 3 & 80 a $150 \mathrm{mg} / \mathrm{dia}$ \\
\hline & 4 a 8 & 100 a $200 \mathrm{mg} / \mathrm{dia}$ \\
\hline & $>8$ & 200 a 400 mg/dia \\
\hline \multirow[t]{4}{*}{ Vitamina $\mathrm{K}$} & $<1$ & - \\
\hline & 1 a 3 & - \\
\hline & 4 a 8 & 0,3 a $0,5 \mathrm{mg} / \mathrm{dia}$ \\
\hline & $>8$ & - \\
\hline \multirow[t]{5}{*}{ Sódio } & 0 a 6 meses & $90 \mathrm{mg} / \mathrm{kg} / \mathrm{dia}$ \\
\hline & 7 a 12 meses & $45 \mathrm{mg} / \mathrm{kg} / \mathrm{dia}$ \\
\hline & 1 a 5 anos & $0,5 \mathrm{~g} / \mathrm{dia}$ \\
\hline & 6 a 10 anos & 1g/dia \\
\hline & $>10$ anos & 1,5 a $2 \mathrm{~g} / \mathrm{dia}$ \\
\hline Cálcio & Crianças & 400 a $800 \mathrm{mg}$ \\
\hline Ferro & Crianças & 5 a $10 \mathrm{mg} / \mathrm{dia}$ \\
\hline Zinco & Crianças & 5 a $10 \mathrm{mg} / \mathrm{dia}$ \\
\hline
\end{tabular}

UI: unidade internacional 
O Consenso Europeu sobre Nutrição em FC sugere medir as concentrações plasmáticas de vitaminas lipossolúveis pelo menos uma vez por ano e sempre que haja alterações no tratamento de reposição vitamínica ou de má absorção, especialmente em pacientes com insuficiência pancreática ${ }^{(3,10)}$.

As vitaminas hidrossolúveis parecem ter boa absorção, com exceção da vitamina $B_{12}$. Com a suplementação de enzimas pancreáticas, a vitamina $\mathrm{B}_{12}$ é normalmente absorvida, exceto quando há ressecção extensa do íleo terminal; neste caso, a reposição é mensal por via parenteral ${ }^{(3,10)}$. Sua deficiência resulta em anemia megaloblástica, neuropatia, ateroma e esteatose hepática ${ }^{(46)}$.

Com relação aos minerais e elementos traço, pacientes com FC apresentam risco de hiponatremia devido à perda de sal, principalmente pelo suor durante períodos de calor, febre e exercício físico. Por isso, é recomendada a adição de sal às refeições. Os mecanismos compensatórios da supra-renal tornam a hiponatremia geralmente assintomática. A suplementação de sódio é realizada segundo a faixa etária (Tabela 4) ${ }^{(40)}$.

A baixa da ingestão e/ou má absorção de cálcio é um dos vários fatores envolvidos na patogênese da osteoporose. Assim, a oferta de cálcio e a exposição ao sol devem ser orientados em idade precoce ${ }^{(47)}$. Sua suplementação oral é indicada quando a alimentação não fornecer o aporte recomendado, que varia de acordo com a da idade e velocidade de crescimento (Tabela 4$)^{(47)}$.

A deficiência de ferro é frequente na FC, sendo causada por múltiplos fatores: ingestão inadequada, má absorção, infecção crônica e perda sanguínea. A recomendação para crianças é de 5 a 10mg/dia (Tabela 4) e sua administração medicamentosa não deve ocorrer ao mesmo tempo que a da enzima pancreática. A necessidade da suplementação deve ser determinada pela análise da saturação de transferrina plasmática ${ }^{(40)}$.

O zinco é importante para o funcionamento de várias enzimas. A sua deficiência é caracterizada pelo retardo do crescimento, acrodermatite e distúrbios na função imune. A recomendação para crianças é de 5 a $10 \mathrm{mg} / \mathrm{dia}(\text { Tabela } 4)^{(3)}$. A má absorção de gordura favorece a perda fecal de zinco, por

\section{Referências bibliográficas}

1. Boat TF. Fibrose cística. In: Behrman RE, Kliegman RM, Jenson HB. Nelson - Tratado de Pediatria 15 ed. Rio de Janeiro: Guanabara Koogan, 2000.

2. Brennan AL, Geddes DM. Cystic fibrosis. Curr Opin Infect Dis 2002; 15:175-82

3. Sinaasappel M, Stern M, Littlewood J, Wolfe S, Steinkamp G, Heijerman HG et al. Nutrition in patients with cystic fibrosis: a European Consensus. J Cyst Fibros 2002;1:51-75. isso a suplementação enzimática aumenta a sua absorção ${ }^{(48)}$, mesmo assim a sua reposição deve ser rotineira.

\section{Diabetes relacionado à fibrose cística}

Cerca de 10 a $25 \%$ dos pacientes com FC desenvolvem diabetes melito (DM), cuja patogênese está associada com fibrose e destruição pancreática, sendo mais frequente em indivíduos que apresentam insuficiência pancreática exócrina. A dieta para pacientes fibrocísticos com e sem diabetes é semelhante com relação à distribuição, aporte energético e fracionamento, habitualmente em seis refeições. Os açúcares simples não devem ser restritos quando necessários para alcançar os requerimentos energéticos, mas as doses de insulina devem ser ajustadas ${ }^{(49)}$.

Pacientes com intolerância à glicose e com diabetes relacionado à $\mathrm{FC}$ apresentam aumento do estresse oxidativo e, por isso, devem ser considerados os nutrientes antioxidantes no tratamento ${ }^{(49)}$.

\section{Considerações finais}

A perda de peso é reconhecida como o pior marcador no prognóstico da $\mathrm{FC}$ e as deficiências nutricionais podem ocorrer em idade precoce. Por isso, a avaliação do estado nutricional e a orientação alimentar devem ser realizadas assim que estabelecido o diagnóstico da doença.

O crescimento normal pode ser alcançado na maioria dos pacientes com dieta hipercalórica, sem restrição lipídica e reposição adequada de enzimas pancreáticas. No caso do paciente não conseguir atender às necessidades nutricionais com o plano alimentar, deve-se indicar a suplementação por via oral e/ou a terapia nutricional (sondas ou ostomias). Desta forma, o monitoramento nutricional e o aconselhamento dietético são elementos chave no manejo de crianças e adolescentes com FC com o intuito de controlar a sintomatologia e retardar a progressão da doença, proporcionando melhor qualidade de vida.

4. Lai HJ. Classification of nutritional status in cystic fibrosis. Curr Opin Pulm Med 2006;12:422-7.

5. Corey M, McLaughlin FJ, Williams M, Levison H. A comparison of survival, growth, and pulmonary function in patients with cystic fibrosis in Boston and Toronto. J Clin Epidemiol 1988;41:583-91.

6. Pencharz PB, Durie PR. Pathogenesis of malnutrition in cystic fibrosis, and its treatment. Clin Nutr 2000;19:387-94. 
7. Groeneweg M, Tan S, Boot AM, de Jongste JC, Bouquet J, Sinaasappel M. Assessment of nutritional status in children with cystic fibrosis: conventional anthropometry and bioelectrical impedance analysis. A cross-sectional study in Dutch patients. J Cyst Fibros 2002;1:276-80.

8. Zemel BS, Jawad AF, FitzSimmons S, Stallings VA. Longitudinal relationship among growth, nutritional status, and pulmonary function in children with cystic fibrosis: analysis of the Cystic Fibrosis Foundation National CF Patient Registry. J Pediatr 2000;137:374-80.

9. Borowitz D, Baker RD, Stallings V. Consensus report on nutrition for pediatric patients with cystic fibrosis. J Pediatr Gastroenterol Nutr 2002;35:246-59.

10. Stallings VA, Stark LJ, Robinson KA, FeranchakAP, Quinton H; Clinical Practice Guidelines on Growth and Nutrition Subcommitee et al. Evidence-based practice recommendations for nutrition-related management of children and adults with cystic fibrosis and pancreatic insufficiency: results of a systematic review. J Am Diet Assoc 2008;108:832-9.

11. Allen JR, McCauley JC, Selby AM, Waters DL, Gruca MA, Baur LA et al. Differences in resting energy expenditure between male and female children with cystic fibrosis. J Pediatr 2003;142:15-9.

12. Koletzko $S$, Reinhardt $D$. Nutritional challenges of infants with cystic fibrosis. Early Hum Dev 2001;65 (Suppl):S53-61.

13. Anthony $\mathrm{H}$, Bines J, Phelan P, Paxton S. Relation between dietary intake and nutritional status in cystic fibrosis. Arch Dis Child 1998;78:443-7.

14. Elborn JS, Cordon SM, Western PJ, Macdonald IA, Shale DJ. Tumour necrosis factor-alpha, resting energy expenditure and cachexia in cystic fibrosis. Clin Sci (Lond) 1993;85:563-8.

15. Adde FV, Dolce P, Tanikawa CE, Uehara DY, Cardoso AL, Rosov T. Nutritional supplementation in patients with cystic fibrosis. J Pediatr (Rio J) 1997;73:317-23.

16. Littlewood JM, Wolfe SP. Control of malabsorption in cystic fibrosis. Paediatr Drugs 2000;2:205-22.

17. Sharma M, Singh M. Nutritional management of children with cystic fibrosis. Indian Pediatr 2003;40:1055-62.

18. Roulet M, Frascarolo P, Rappaz I, Pilet M. Essential fatty acid deficiency in well nourished young cystic fibrosis patients. Eur J Pediatr 1997;156:952-6.

19. Parsons HG, O'Loughlin EV, Forbes D, Cooper D, Gall DG. Supplemental calories improve essential fatty acid deficiency in cystic fibrosis patients. Pediatr Res 1988;24:353-6.

20. Freedman SD, Blanco PG, Zaman MM, Shea JC, Ollero M, Hopper IK et al. Association of cystic fibrosis with abnormalities in fatty acid metabolism. $\mathrm{N}$ Engl J Med 2004;350:560-9.

21. Steinkamp G, Wiedemann B. Relationship between nutritional status and lung function in cystic fibrosis: cross sectional and longitudinal analyses from the German CF quality assurance (CFQA) project. Thorax 2002;57:596-601.

22. Colombo C, Bennato V, Costantini D, Valmarana L, Daccò V, Zazzeron L et al. Dietary and circulating polyunsaturated fatty acids in cystic fibrosis: are they related to clinical outcomes? J Pediatr Gastroenterol Nutr 2006;43:660-5.

23. Al-Turkmani MR, Andersson C, Alturkmani R, Katrangi W, Cluette-Brown JE, Freedman SD et al. A mechanism accounting for the low cellular level of linoleic acid in cystic fibrosis and its reversal by DHA. J Lipid Res 2008;49:1946-54.

24. Shoff SM, Ahn HY, Davis L, Lai H; Wisconsin CF Neonatal Screening Group. Temporal associations among energy intake, plasma linoleic acid, and growth improvement in response to treatment initiation after diagnosis of cystic fibrosis. Pediatrics 2006;117:391-400.

25. De Vizia B, Raia V, Spano C, Pavlidis C, Coruzzo A, Alessio M. Effect of an 8-month treatment with omega-3 fatty acids (eicosapentaenoic and docosahexaenoic) in patients with cystic fibrosis. J Parenter Enteral Nutr 2003;27:52-7.

26. Wood LG, Fitzgerald DA, Gibson PG, Cooper DM, Garg ML. Increased plasma fatty acid concentrations after respiratory exacerbations are associated with elevated oxidative stress in cystic fibrosis patients. Am J Clin Nutr 2002;75:668-75.

27. Panchaud A, Sauty A, Kernen Y, Decosterd LA, Buclin T, Boulat O et al. Biological effects of a dietary omega- 3 polyunsaturated fatty acids supplementation in cystic fibrosis patients: a randomized, crossover placebocontrolled trial. Clin Nutr 2006;25;418-27.

28. McKarney C, Everard M, N'Diaye T. Omega-3 fatty acids (from fish oils) for cystic fibrosis. Cochrane Database Syst Rev 2007;17(4):CD002201.

29. Ramsey BW, Farrell PM, Pencharz P. Nutritional assessment and management in cystic fibrosis: a consensus report. The Consensus Committee. Am J Clin Nutr 1992;55:108-16.

30. Joint $F A O / W M O / U N U$ Expert Consultation. Energy and protein requirements. Technical report $n^{\circ} 724$. Geneva: WHO; 1985.

31. Wilson DC, Pencharz PB. Nutrition in cystic fibrosis. Nutrition. 1998;14(10):792-795.

32. Rosenfeld M, Casey S, Pepe M, Ramsey BW. Nutritional effects of long-term gastrostomy feedings in children with cystic fibrosis. J Am Diet Assoc. 1999; 99(2):191-4

33. Oliveira G, Oliveira C. Nutrition, cystic fibrosis and the digestive tract. Nutr Hosp 2008;23 (Suppl 2):71-86.

34. Figueroa V, Milla C, Parks EJ, Schwarzenberg SJ, Moran A. Abnormal lipid concentrations in cystic fibrosis. Am J Clin Nutr 2002;75:1005-11.

35. Serra L, Aranceta J. Objetivos nutricionales para la población española: consenso de la sociedad española de nutrición comunitaria (SENC). In: Aranceta J. Guías alimentarias para la población española. Madrid: IM\&C; 2001. p. 345-51

36. Institute of Medicine of The National Academies. Food and Nutrition Board. Dietary Reference Intakes for Energy, Carbohydrate. Fiber, Fat, Fatty Acids, Cholesterol, Protein, and Amino Acids (2002/2005). [cited 2011 Dec 12]. Available from: http://www.nap.edu/openbook.php

37. Powers SW, Patton SR, Byars KC, Mitchell MJ, Jelalian E, Mulvihill MM et al. Caloric intake and eating behavior in infants and toddlers with cystic fibrosis. Pediatrics 2002;109:E75-5.

38. McDonald CM. Validation of a nutrition risk screening tool for children and adolescents with cystic fibrosis ages 2-20 years. J Pediatr Gastroenterol Nutr 2008;46:438-46.

39. Dodge JA, Turck D. Cystic fibrosis: nutritional consequences and management. Best Pract Res Clin Gastroenterol 2006;20:531-46.

40. Arias MM, Bozano GP, Osés JS, Allué IP. Fibrosis quística: aspectos nutricionales. An Pediatr (Barc) 2001;54:575-81.

41. Poustie VJ, Russell JE, Watling RM, Ashby D, Smyth RL; CALICO Tria Collaborative Group. Oral protein energy supplements for children with cystic fibrosis: CALICO multicentre randomised controlled trial. BMJ 2006;332:632-6

42. Bell SC, Shepherd RW. Optimising nutrition in cystic fibrosis. J Cyst Fibros 2002;1:47-50.

43. Wood LG, Fitzgerald DA, Gibson PG, Cooper DM, Collins CE, Garg ML. Oxidative stress in cystic fibrosis: dietary and metabolic factors. J Am Coll Nutr 2001;20 (Suppl 2):157-65.

44. MacDonald A. The diet in cystic fibrosis: why is it important? Curr Paediatr 2000;10:155-61

45. Hakim F, Kerem E, Rivlin J, Bentur L, Stankiewicz H, Bdolach-Abram T et al Vitamins $A$ and $E$ and pulmonary exacerbations in patients with cystic fibrosis. J Pediatr Gastroenterol Nutr 2007;45:347-53.

46. Weir DG, Scott JM. Vitamina $B_{12}$ "Cobalamina". In: Shils ME, Olson JA, Shike M, Ross AC, editors. Tratado de nutrição moderna na saúde e na doença. 9 ed. São Paulo: Manole; 2003. p.477-88.

47. Lambert JP. Osteoporosis: a new challenge in cystic fibrosis. Pharmacotherapy 2000;20:34-51.

49. Krebs NF, Sontag M, Accurso FJ, Hambidge KM. Low plasma zinc concentrations in young infants with cystic fibrosis. J Pediatr 1998;133:761-4.

49. American Diabetes Association, Bantle JP, Wylie-Rosett J, Albright AL, Apovian $\mathrm{CM}$, Clark NG et al. Nutrition recommendations and interventions for diabetes: a position statement of the American Diabetes Association. Diabetes Care 2008;31 (Suppl 1):S61-78. 